\title{
DEBATE
}

\section{Pro: Alzheimer's disease and circadian dysfunction: chicken or egg?}

\author{
Tracy A Bedrosian* and Randy J Nelson
}

\begin{abstract}
Alzheimer's disease (AD) is a neurodegenerative disease that accounts for most cases of dementia. Besides progressive cognitive decline, circadian dysfunction is a prominent feature of AD. Circadian disruption is traditionally regarded as a downstream symptom of $A D$, but recent evidence suggests that circadian dysregulation may act to exacerbate AD pathology. A reciprocal link among sleep, circadian rhythms, and amyloid deposition has long been suspected, and data from both human and animal studies support this hypothesis. The sleep-wake cycle regulates amyloid-beta (A $\beta$ ) levels in both mice and humans. Sleep deprivation increases $A \beta$ levels in mice, and sleep apnea and insomnia may be related to $A D$ in humans. Furthermore, melatonin, the principal hormonal output of the circadian system, is dysregulated in $A D$, and this may be important because melatonin is protective in cells exposed to toxic $A \beta$. Initial evidence supports a reciprocal link among sleep, circadian rhythmicity, and AD. More investigation is necessary to replicate these studies and determine the extent to which the circadian system contributes to the pathogenesis of AD.
\end{abstract}

\section{Background}

Alzheimer's disease (AD) is a neurodegenerative disease that accounts for most cases of dementia. Accumulation and aggregation of amyloid-beta $(A \beta)$ peptide in the brain extracellular space are main features of $\mathrm{AD}$ pathogenesis. $A \beta$ is secreted by neurons into the brain interstitial fluid (ISF). In $A D$, however, excessive $A \beta$ accumulates and aggregates into plaques, which contribute to progressive cognitive deficits. Circadian dysfunction is another prominent feature of $\mathrm{AD}$, and recent evidence suggests

*Correspondence: tracy.bedrosian@osumc.edu

Department of Neuroscience, The Ohio State University Wexner Medical Center, 333 West 10th Avenue, Columbus, OH 43210, USA that $\mathrm{AD}$ pathology, in turn, may be exacerbated by circadian dysregulation.

Aging is associated with a host of changes to the circadian organization of physiological and behavioral rhythms, but these changes are highly pronounced in AD. For example, reduced amplitude of 24-hour rhythms in arousal, body temperature, and hormone fluctuations is typical among older individuals (reviewed in [1]). Altered period and responsiveness to zeitgebers (that is, entraining cues) are observed; furthermore, increasing circadian disorganization is associated with the severity of the AD pathology [2].

Circadian dysfunction is implicated in the pathology of several other disease states as well. For example, major depression may be associated with sleep disruption, diurnal mood swings, and elevated nocturnal body temperature; manipulation of sleep and the circadian cycle is an effective, though short-lasting, treatment. Manipulation of the circadian light-dark cycle causes direct changes to brain structure and function [3]. Seasonal affective disorder, also known as winter depression, is directly related to light and the circadian system. A phase delay in the melatonin rhythm is thought to partially underlie this disorder (reviewed in [4]).

Clearly, AD is associated with circadian dysfunction, but the extent to which these changes constitute symptoms, disease pathogenesis, or both remains unspecified. This article will present evidence in support of a reciprocal relationship between the circadian system and AD pathology.

\section{Discussion}

A reciprocal link among sleep, circadian rhythms, and amyloid deposition has long been suspected on the basis of an early observation that sleep apnea appeared to be highly prevalent among patients with AD [5]. Despite a small sample size in that study, subsequent data from patients with sleep apnea demonstrated an association with the apolipoprotein E4 (APOE4) genotype [6] and improvements in dementia symptoms after continuous positive airway pressure treatment $[7,8]$. Furthermore, serum amyloid A levels tend to be increased in patients with sleep apnea, leading potentially to the eventual 
formation of amyloid fibrils and thus contributing to AD pathology [9], but the role of amyloid A in accelerating $\mathrm{A} \beta$ deposition in plaques remains unclear. Alternatively, obstructive sleep apnea may worsen cognition via vascular or hypoxemic mechanisms that are unrelated to circadian disruption. More work is necessary to understand whether data on sleep apnea support a circadian hypothesis of AD.

Recently, direct evidence from both animals and humans has emerged to support the notion that $A \beta$ dynamics are regulated by the sleep-wake cycle. Using transgenic mice expressing a mutated form of the human amyloid precursor protein, Holtzman's group [10] observed that a diurnal rhythm in brain ISF A $\beta$ levels was negatively correlated with the amount of time spent asleep. Sleep deprivation during the inactive phase increased ISF A $\beta$ levels, as did infusion of orexin- $A$ at a dose sufficient to promote wakefulness. A dual orexin receptor antagonist, on the other hand, reduced $A \beta$ and abolished the diurnal rhythm in $\mathrm{A} \beta$ levels. Furthermore, $\mathrm{A} \beta$ plaque deposition in the brain was enhanced by chronic sleep restriction and also was ameliorated after treatment with the orexin receptor antagonist [10]. This study provided direct evidence that sleep and the circadian system may have a role in the pathogenesis of AD.

Since these observations were first reported in mice, the findings have been replicated in a cohort of human patients with $\mathrm{AD}$. $\mathrm{A} \beta$ measurements in human cerebrospinal fluid revealed age-related disruptions in the normal circadian pattern and a negative correlation with the amount of sleep received [11]. Furthermore, a longitudinal study evaluating older adults for almost 8 years showed that chronic insomnia was associated with AD. Patients beginning the study with normal cognition and insomnia progressed more quickly to dementia in comparison with those without insomnia [12]. This study provides evidence in humans that chronic sleep-wake disturbance could increase the risk of developing AD.

Neuronal activity may be responsible for regulating $A \beta$ dynamics. Brain ISF A $\beta$ concentrations are closely related to levels of ISF lactate, a marker of neuronal activity. Changes in neuronal activity, in turn, modulate both ISF $\mathrm{A} \beta$ and amyloid plaque formation [13]. As sleep is associated with a net decrease in synaptic strength whereas wakefulness is associated with a net increase [14], the circadian sleep-wake cycle may act to modulate $\mathrm{A} \beta$ levels via changes in neuronal activity. In this case, it is plausible that circadian disruptions or sleep disturbances could contribute to AD pathogenesis over the long term by altering neuronal activity patterns.

Melatonin is a principal hormonal output of the circadian system. It is produced by the pineal gland in a rhythmic pattern in response to the daily light-dark cycle and its secretion is regulated via the suprachiasmatic nuclei. Melatonin secretion tends to be reduced among older individuals but even more so among patients with dementia [15]. This circadian disruption of the melatonin system provoked by dementia may have physiological effects on AD pathology. For example, in vitro evidence suggests that melatonin prevents the death of neuroblastoma cells exposed to $A \beta$ [16]. Clinical trials investigating the effects of melatonin on cognition have not produced clear results, however. One trial in patients with AD showed no improvement in cognition and behavior and only small effects on sleep quality [17], whereas a small trial in patients with mild cognitive impairment suggested improved cognitive performance [18].

\section{Summary}

Although much of the research discussed here specifically relates sleep, rather than circadian rhythms in general, to AD pathology, it is important to bear in mind that circadian dysfunction is often upstream of any sleep disorder. The Borbély two-process model of sleep regulation proposes both sleep-dependent and sleepindependent processes that influence the propensity to sleep [19]. On one hand, the drive to sleep is a function of prior wake time. On the other hand, sleep is a function of underlying homeostatic drive controlled by endogenous circadian oscillators. These independent processes need to be studied both in isolation and together to fully understand the impact of circadian organization and sleep on disease and health.

Both sleep and circadian dysfunction are closely associated with $\mathrm{AD}$, and given the role of the circadian system in promoting or exacerbating other disease states, it is plausible that circadian disturbance could contribute to the pathogenesis of $\mathrm{AD}$ as well [20]. More research is necessary to replicate and expand upon the finding that sleep-wake cycles regulate $A \beta$, but converging evidence from sleep disorders (for example, sleep apnea) and melatonin research support the notion that sleep and circadian rhythmicity may have a reciprocal link to AD pathology.

Abbreviations

$A \beta$, amyloid-beta; AD, Alzheimer's disease; ISF, interstitial fluid.

Competing interests

The authors declare that they have no competing interests.

Published: 13 August 2012

\section{References}

1. Van Someren EJ: Circadian and sleep disturbances in the elderly. Exp Gerontol 2000, 35:1229-1237.

2. Hatfield CF, Herbert J, van Someren EJ, Hodges JR, Hastings MH: Disrupted daily activity/rest cycles in relation to daily cortisol rhythms of homedwelling patients with early Alzheimer's dementia. Brain 2004, 127 (Pt 5):1061-1074 
3. Bedrosian TA, Weil ZM, Nelson RJ: Chronic dim light at night provokes reversible depression-like phenotype: possible role for TNF. Mol Psychiatry, in press.

4. Bunney WE, Bunney BG: Molecular clock genes in man and lower animals: possible implications for circadian abnormalities in depression. Neuropsychopharmacology 2000, 22:335-345.

5. Smirne S, Franceschi M, Bareggi SR, Comi G, Mariani E, Mastrangelo M: Sleep Apneas in Alzheimer's Disease. Edited by Koella WP. Amsterdam: 5th European Congress of Sleep Research; 1980:442-444.

6. Kadotani H, Kadotani T, Young T, Peppard PE, Finn L, Colrain IM, Murphy GM Jr., Mignot E: Association between apolipoprotein E epsilon4 and sleepdisordered breathing in adults. JAMA 2001, 285:2888-2890.

7. Ancoli-Israel S, Palmer BW, Cooke JR, Corey-Bloom J, Fiorentino L, Natarajan L, Liu L, Ayalon L, He F, Loredo JS: Cognitive effects of treating obstructive sleep apnea in Alzheimer's disease: a randomized controlled study. J Am Geriatr Soc 2008, 56:2076-2081

8. Bliwise DL: Sleep apnea, APOE4 and Alzheimer's disease 20 years and counting? J Psychosom Res 2002, 53:539-546.

9. Svatikova A, Wolk R, Shamsuzzaman AS, Kara T, Olson EJ, Somers VK: Serum amyloid a in obstructive sleep apnea. Circulation 2003, 108:1451-1454.

10. Kang JE, Lim MM, Bateman RJ, Lee JJ, Smyth LP, Cirrito JR, Fujiki N, Nishino S, Holtzman DM: Amyloid-beta dynamics are regulated by orexin and the sleep-wake cycle. Science 2009, 326:1005-1007.

11. Huang Y, Potter R, Sigurdson W, Santacruz A, Shih S, Ju YE, Kasten T, Morris JC, Mintun M, Duntley S, Bateman RJ: Effects of age and amyloid deposition on Abeta dynamics in the human central nervous system. Arch Neurol 2012, 69:51-58.

12. Osorio RS, Pirraglia E, Agüera-Ortiz LF, During EH, Sacks H, Ayappa I, Walsleben J, Mooney A, Hussain A, Glodzik L, Frangione B, Martínez-Martín P, de Leon $\mathrm{MJ}$ : Greater risk of Alzheimer's disease in older adults with insomnia. J Am Geriatr Soc 2011, 59:559-562.

13. Bero AW, Yan P, Roh JH, Cirrito JR, Stewart FR, Raichle ME, Lee JM, Holtzman DM: Neuronal activity regulates the regional vulnerability to amyloid-beta deposition. Nat Neurosci 2011, 14:750-756.
14. Vyazovskiy W, Cirelli C, Pfister-Genskow M, Faraguna U, Tononi G: Molecular and electrophysiological evidence for net synaptic potentiation in wake and depression in sleep. Nat Neurosci 2008, 11:200-208.

15. Souetre E, Salvati E, Krebs B, Belugou JL, Darcourt G: Abnormal melatonin response to 5-methoxypsoralen in dementia. Am J Psychiatry 1989, 146:1037-1040.

16. Pappolla MA, Sos M, Omar RA, Bick RJ, Hickson-Bick DL, Reiter RJ, Efthimiopoulos S, Robakis NK: Melatonin prevents death of neuroblastoma cells exposed to the Alzheimer amyloid peptide. J Neurosci 1997, 17:1683-1690.

17. Singer C, Tractenberg RE, Kaye J, Schafer K, Gamst A, Grundman M, Thomas R, Thal L; Alzheimer's Disease Cooperative Study: A multicenter, placebocontrolled trial of melatonin for sleep disturbance in Alzheimer's disease. Sleep 2003, 26:893-901.

18. Peck JS, LeGoff DB, Ahmed I, Goebert D: Cognitive effects of exogenous melatonin administration in elderly persons: a pilot study. Am J Geriatr Psychiatry 2004, 12:432-436.

19. Borbely AA, Achermann P: Sleep homeostasis and models of sleep regulation. J Biol Rhythms 1999, 14:557-568.

20. Slats $D$, Claassen JA, Verbeek MM, Overeem S: Reciprocal interactions between sleep, circadian rhythms and Alzheimer's disease: focus on the role of hypocretin and melatonin. Ageing Res Rev 2012 Apr 30. [Epub ahead of print].

doi:10.1186/alzrt128

Cite this article as: Bedrosian TA, Nelson RJ: Pro: Alzheimer's disease and circadian dysfunction: chicken or egg? Alzheimer's Research \& Therapy 2012, 4:25 